\title{
Enrollment of children in cardiac critical care clinical trials
}

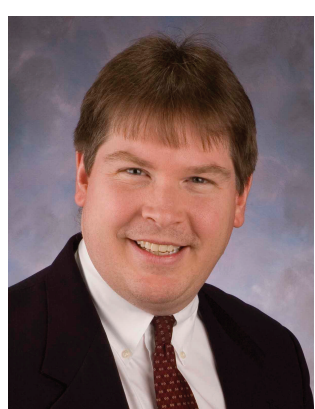

Timothy M Hoffman Nationwide Children's Hospital, Division of Cardiology, Room 616ED, 700 Children's Drive, Columbus, OH 43205, USA Tel.: + 1614722 2530; Fax: +1 614722 2549; E-mail: timothy.hoffman@ nationwidechildrens.org

\section{'Grant support has decreased over the last several years and industry- sponsored trials often need financial motivation to augment the process of trial development and execution.'}

Pediatric cardiac critical care trials are essential to the development of safe and effective management strategies for children with either congenital or acquired heart disease. According to the American Heart Association (AHA), nearly 40,000 babies are born each year with a form of congenital heart disease. There are well over 35 forms of congenital heart defects that can be extremely complex in nature and physiology. Inter-disciplinary teams of physicians with varying backgrounds care for these infants and children throughout their lives. An increasing number of patients are living longer after surgical or catheter-based intervention and, therefore, patients are transitioned to adult congenital heart disease experts for their care, to assure adequate management of the consequences of congenital heart defects with time. Consequently, many patients receive care recurrently in the Cardiac Intensive Care Unit (CICU), which is a specialized unit committed to the critical care of patients with congenital or acquired heart disease. As with most of pediatric medicine, cardiac related multicenter trials are a rarity and pose difficult problems in development and execution. For example, only approximately $6 \%$ of the 80 most commonly used medications in infants and children are approved for pediatric use. Additionally, approximately $75 \%$ of all medications on the market do not have approved pediatric labeling. Caregivers choose medications based upon anecdotal experience and preference, extrapolation of adult studies and small studies in the pediatric population that often are not designed appropriately as a randomized, doubleblind, placebo-controlled trial. The pediatric cardiac community depends heavily on professional interactions to establish quality assurance measures and academic advancement. Recently in 2003, the Pediatric Cardiac Intensive Care Society (PCICS) was formed to provide an international professional forum to promote excellence in pediatric cardiac critical care medicine. This society has many goals but certainly centers around symposia, education and the promotion of translational research amongst centers across the world. This society has a diverse membership of physicians, nurses and allied health professionals, all with the common goal of collegially advancing the care for these children.

So why is it so difficult to obtain the data needed to make informed decisions in our patients? The challenge to evidence-based medicine in the field of pediatric cardiology is multifactorial. Some obstacles are simple realities that are difficult to overcome. For instance, in order to demonstrate statistically significant differences between treatment $A$ and treatment $B$ in controlled trials, many patients, often thousands, must be enrolled in the study.

$\therefore$...there are public and medical perceptions that interfere with successful study recruitment.'

While there are 40,000 infants with congenital heart disease born each year, only approximately $35 \%$ require intervention in the first year of life. And these patients are spread across hundreds of academic and nonacademic practices. No single institution caring for these patients is capable of conducting an appropriately powered drug trial in a short enough period of time to allow for practice evolution. Additionally, no common clinical database exists, although attempts have been made to develop registries for certain disease processes. These registries all have limits, which may include funding, staff and scientific inquiries that change with experience and time, thus sometimes making the existing database dated and insufficient to answer contemporary questions. Development of multicenter collaboratives, such as the Pediatric Heart Network through the NIH, is a step in the right direction. Some obstacles are fiscal realities, such as a limited NIH budget, which has stayed static 
for 5 years. Physicians must turn to other sources for funding, such as industry sponsorship. Finally, there are public and medical perceptions that interfere with successful study recruitment. There is a notion that research is fine as long as it does not involve my child or my patient. Why is this? Does the public feel that unnecessary risk is being placed upon study patients? Does enrolling in an industry-sponsored drug trial bypass the protections of the US FDA? Are industry sponsored drug studies unsafe?

'...approximately $75 \%$ of all medications on the market do not have approved pediatric labeling.'

The FDA has enacted new regulations designed to promote privately funded drug trials in children. The FDA Modernization Act (FDAMA) in November of 1997, specifically addressed pediatric drug trials and also provided impetus to sponsors to complete trials by providing 6 months of extended market exclusivity [1]. Under the US Food, Drug and Cosmetic Act in 1999, regulations were set to industry for new drugs anticipated for pediatric use. Not only was early planning of trials for the particular drug encouraged, but a requirement for studying and labeling these drugs for pediatric use was established. The Best Pharmaceuticals for Children Act was placed into law in 2002, which in essence renewed the exclusivity previously enacted in 1997 under FDAMA [2]. This act also detailed public posting of results of trials and assured reporting of adverse events for 1 year after exclusivity was granted. In 2003, the Pediatric Research Equity Act provided the FDA the authority to require the study of drugs for the pediatric population to assure safety [3]. Most recently, the Pediatric Research Equity Act of 2007 has established a Pediatric Review Committee to help evaluate written requests for studies and to be a public liaison for dissemination of information concerning changes in labeling or pediatric studies. Despite these governmental acts, pediatric cardiac critical care trials are still uncommon.

Based upon personal experience, industry sponsors are extremely willing to discuss clinical trials when there are drugs that are new to the pediatric market and drugs that are reaching the end of the patent. The largest pediatric cardiac critical care trial to date, PRIMACORP [4,5], was successful owing to an exquisite relationship between industry and clinical leaders in pediatric cardiac critical care, however, this project benefited greatly in that milrinone was to become generic. The 6-month exclusivity clause of the aforementioned governmental acts was a financial motivation for the sponsor that allowed for excellent research collaboration and a successful clinical trial. Attempting to recreate that type of system has been difficult, even with medicines that are similar in effect to milrinone, as many sponsors seek overseas trials which are governed differently. Grant support has decreased over the last several years and industry sponsored trials often need financial motivation to augment the process of trial development and execution. This may pose a barrier to the successful completion of much needed research in this field.

Of utmost importance, the patient must be kept safe and the parents must be comfortable once they are approached or enrolled in a trial. Institutional Review Boards are in place to assure protection and the rights of human subjects. In other words, these boards function as a so-called ethics committee for research and are present in any institution that conducts research. Any researcher, regardless of training or background, must adhere to the Belmont Report, which encompasses three principles, namely respect for persons, beneficence and justice.

'It is ... imperative that a coordinated
approach of all responsible researchers
assures that the family is well informed
and the consent process is without bias.'

Pediatric cardiac trial design may be impacted by the heterogeneous patient population with a multitude of underlying cardiac defects. Additionally, many patients receive a surgical intervention that may elicit a strong parental emotional response at the time of potential trial enrollment. In a recent study, a voluntary, selfadministered questionnaire was provided to parents of children 16 years of age or less during the preadmission testing period a day prior to their child's surgical procedure. This study examined parental views of several key elements to pediatric cardiac critical care research [6]. Basic knowledge and experience with research was limited. Very few respondents had their child $(8 \%)$ or knew of someone $(11 \%)$ that had participated in a clinical trial previously. Almost two-thirds of the respondents felt that most medicines used in pediatrics were approved by the FDA for use in children. Despite this lack of experience and misunderstanding of 
FDA-approved medications, the overwhelming majority $(91 \%)$ of respondents felt that clinical trials will help improve pediatric care, and $74 \%$ felt that by enrolling their child in a trial, the participant may receive a medicine or device that could be of benefit.

Clinical trial design may also be facilitated by a medicine or device that is already approved in adults or one that is used in clinical practice by their physician. In the aforementioned questionnaire study [6], 50\% of the parents felt more comfortable enrolling their child in a trial that involves a drug or device currently used in their physician's practice, whereas $19 \%$ were encouraged by a drug or device approved in adults. The majority of parents felt that being approached about a clinical trial by their child's primary pediatric cardiologist or cardiothoracic surgeon was most desirable as opposed to being approached by the principal investigator or the research coordinator.

'Further education about pediatric medicine, drug approval and research is appropriate to all patient families to augment the process at all levels.'

Comfort likely plays a significant role in this process, however, as with any study, one has to guard against coercion. It is thus imperative that a coordinated approach of all responsible researchers assures that the family is well informed and the consent process is without bias. The primary cardiologist or cardiothoracic surgeon will have to be responsible and familiar with all protocols if they are to approach families concerning clinical trials in an unbiased and informative manner. This survey concluded that through appropriate education of parents via proactive and comfortable communication in an appropriate venue, cardiac critical care trials in children may be facilitated even during emotionally stressful hospitalizations. In short, enrollment in pediatric cardiac critical care trials is likely impacted by the type of medicine or device being evaluated, the comfort of the parent, the individuals that approach the family for consent and the emotional factors of having a child in a critical care setting in which much is unknown.

Through governmental acts and the will of expert physicians in pediatric cardiac critical care medicine, clinical trials in this setting will continue to be a focus to improve pediatric care. Ideally, through grant support and industry sponsorship, these trials can be of the utmost quality and design to afford appropriate enrollment, safety and execution. Hopefully, government acts will be an incentive to industry to enlist pediatric clinical experts to augment research and development. Further education about pediatric medicine, drug approval and research is appropriate to all patient families to augment the process at all levels.

\section{Financial \& competing interests disclosure}

The author has no relevant affiliations or financial involvement with any organization or entity with a financial interest in or financial conflict with the subject matter or materials discussed in the manuscript. This includes employment, consultancies, honoraria, stock ownership or options, expert testimony, grants or patents received or pending, or royalties.

No writing assistance was utilized in the production of this manuscript.

\section{Bibliography}

1. Food and Drug Administration Modernization Act of 1997. Public Law 105-115 (1997).

2. Best Pharmaceuticals for Children Act. Public Law 107-109 (2002).

3. Pediatric Research Equity Act of 2003. Public Law 108-155 (2003).
4. Hoffman TM, Wernovsky G, Atz AM et al: Trial Designs: prophylactic intravenous use of milrinone after cardiac operation in pediatrics (PRIMACORP study). Am. Heart J. 143(1), 15-21 (2002).

5. Hoffman TM, Wernovsky G, Atz AM et al: The efficacy and safety of milrinone in preventing low cardiac output syndrome in infants and children after corrective surgery for congenital heart disease. Circulation 107, 996-1002 (2003).

6. Hoffman TM, Taeed R, Niles JP, McMillin MA, Perkins LA, Feltes TF: Parental factors impacting the enrollment of children in cardiac critical care clinical trials. Pediatr. Cardiol. 8(3), 167-171 (2007). 